\title{
Overexpression of SSAT by DENSPM treatment induces cell detachment and apoptosis in glioblastoma
}

\author{
YE TIAN $^{1,2}$, SHIZHAO WANG ${ }^{1,2}$, BIN WANG $^{1}$, JIANNING ZHANG $^{1}$, \\ RONGCAI JIANG ${ }^{1}$ and WEI ZHANG ${ }^{3}$
}

\begin{abstract}
${ }^{1}$ Department of Neurosurgery, Tianjin Medical University General Hospital, Tianjin Neurological Institute, Key Laboratory of Post-trauma Neuro-repair and Regeneration in Central Nervous System, Ministry of Education, Tianjin Key Laboratory of Injuries, Variations and Regeneration of Nervous System, Tianjin; ${ }^{2}$ Tianjin Medical University, Tianjin, P.R. China; ${ }^{3}$ Department of Pathology, The University of Texas, MD Anderson Cancer Center, Houston, TX, USA
\end{abstract}

Received October 2, 2011; Accepted November 23, 2011

DOI: $10.3892 /$ or.2011.1592

\begin{abstract}
N}^{1}, \mathrm{~N}^{11}$-diethylnorspermine (DENSPM), a polyamine analog that induces expression of spermidine/spermine $\mathrm{N}^{1}$-acetyltransferase (SSAT) and reduces polyamine levels in eukaryotic cells, has demonstrated anticancer effects in many cancer cell types. Gene expression of SSAT after treatment with DENSPM was measured in both U87 and LN229 cells using real-time PCR. Induction of SSAT mRNA using DENSPM resulted in significantly higher levels in U87 cells than in LN229 cells. Furthermore, DENSPM caused marked cell detachment in U87 cells and to a lesser extent in LN229 cells. We hypothesized that elevated SSAT expression plays a key role in DENSPM-induced cell detachment in glioblastoma cells. To investigate whether forced expression of SSAT would lead to reduced cell adhesion and increased cell detachment, we transfected a PCMV-SSAT plasmid into LN229 cells and observed significant cell detachment. In addition, we treated U87 cells with SSAT siRNA together with DENSPM to blunt the induction of SSAT by DENSPM. This resulted in an inhibition of cell detachment in U87 cells compared with the DENSPM treatment alone. Increased SSAT expression by transfection enhanced the DENSPM cell-kill effect in LN229 cells whereas reduction of SSAT by siRNA attenuated the DENSPM cell-kill effect. The protein levels of AKT, mTOR and integrin $\alpha 5 \beta 1$, which are members of the cell adhesion and anti-apoptotic signal transduction pathways, were decreased in the PCMV-SSAT transfected LN229 cells. Collectively, these results demonstrate that SSAT induction at least partially plays a role in cell
\end{abstract}

Correspondence to: Dr Rongcai Jiang, Tianjin Neurological Institute, 154 Anshan Road, Heping District, Tianjin 300052, P.R. China

E-mail: jianghope@gmail.com

Key words: $\quad \mathrm{N}^{1}, \mathrm{~N}^{11}$-diethylnorspermine, spermidine/spermine $\mathrm{N}^{1}$-acetyltransferase, glioblastoma, cell detachment, apoptosis detachment and apoptosis of glioblastoma cells by DENSPM treatment.

\section{Introduction}

Polyamines are essential for eukaryotic cell survival although an increase of polyamines has been shown to be associated with survival of cancers. This indicates that the depletion of polyamines may be a potential strategy for cancer treatment. It has long been known that the depletion of polyamines can induce apoptosis in several tumor types using either a polyamine synthesis inhibitor or a polyamine analog $(1,2)$. However, polyamine synthesis inhibitors have only demonstrated a moderate inhibitory effect on glioblastomas (3).

$\mathrm{N}^{1}, \mathrm{~N}^{11}$-diethylnorspermine (DENSPM), a well-studied polyamine analog, was shown in our previous study to induce significant apoptosis in several glioblastoma cell lines (3). Although the mechanism of polyamine analog-induced cell death is still not well understood, it has been shown that DENSPM induces spermidine/spermine acetyltransferase (SSAT) expression, which converts spermine and spermidine to their acetylated forms. Acetylated spermine and spermidine can then serve as substrates for spermine oxidase (SMO) or polyamine oxidase (PAO) in reactions that produce $\mathrm{H}_{2} \mathrm{O}_{2}$ $(4,5)$. In most experiments, DENSPM was described to induce apoptosis in cancer cells by damaging mitochondria, releasing cytochrome c into cytoplasm and activating the caspase- 3 and -8 signal transduction pathways $(6,7)$. We have also observed mTOR relocation in U87 cell lines treated by DENSPM (8).

Unexpectedly, significant cell detachment was observed in some glioblastoma cells but not in others. Consistent with our results, other groups have also reported that marked cell detachment could be induced either in CHENSPM (N1-ethylN11-[(cyclopropyl)methyl]-4,8,-diazaundecane, another polyamine analog) treated lung cancer cells (9) or in DENSPM-treated breast cancer cells (10). Furthermore, the correlation between increased SSAT levels and cell floating in kidney carcinoma cells (HEK293) was also associated with morphological change in cell shape (11). Based on these findings we hypothesized that elevated SSAT expression plays a key role in DENSPM-induced cell detachment in glioblastoma cells. 


\section{Materials and methods}

Cells and cell culture. The human glioblastoma cell lines LN229 and U87 were purchased from American Type Culture Collection (Manassas, VA) and maintained in Dulbecco's modified Eagle's medium (DMEM)/F12 (Gibco, USA) supplemented with $10 \%$ dialyzed serum (Hyclone, Logan, UT) at $37^{\circ} \mathrm{C}$ in a $5 \% \mathrm{CO}_{2}$ incubator. Because fetal bovine serum has abundant thymine and polyamines, we used the dialyzed serum to avoid interfering with the experimental results.

Reagents. DENSPM was purchased from Tocris (Ellisville, $\mathrm{MO}$ ) and was dissolved in water according to the manufacturer's instructions. The PCMV-SSAT and PCMV empty plasmids (negative control) were kindly provided by Professor Eugene Gerner (Arizona Cancer Center, University of Arizona, Tucson, AZ) and were sequenced in our laboratory before using in order to confirm their quality and their human source.

In vitro drug treatment experiments. For the in vitro drug treatment experiments, the cells were seeded in a $10-\mathrm{cm}^{2}$ dish $\left(10^{5}\right.$ cells/dish) in $10 \mathrm{ml}$ of medium supplemented with $10 \%$ dialyzed fetal bovine serum. Twenty-four hours later, $10 \mu \mathrm{M}$ DENSPM was added.

PCMV-SSAT transfection. After LN229 cells reached 80\% confluency in the culture plates, they were collected by trypsinization and counted. The PCMV-SSAT and negative control PCMV empty plasmids were transfected into $1 \times 10^{6}$ LN229 cells in parallel with Nucleofector technology according to the manufacturer's protocol (Amaxa Biosystems, Gaithersburg, MD). Before transfection, a GFP plasmid was added into the target plasmids to serve as the illumination marker to confirm successful transfection. The transfected LN229 cells were distributed into $10-\mathrm{cm}^{2}$ dishes and continuously cultured in the humidified incubator containing $5 \% \mathrm{CO}_{2}$ at $37^{\circ} \mathrm{C}$ for another $24 \mathrm{~h}$.

Knockdown of SSAT expression by siRNA. Dharmacon SMARTpool ${ }^{\circledR}$ siRNAs (Dharmacon, Lafayette, CO) were used for silencing SSAT with Nucleofector technology according to the manufacturer's protocol. For the non-specific target, nonsense siRNA (Ambion Inc., Austin, TX) was used as a control. Briefly, 2-3x10 ${ }^{6}$ LN229 or U87 cells were resuspended in $100 \mu \mathrm{l}$ of Nucleofector solution with $100 \mathrm{nM}$ of siRNA in the electroporation cuvette. After electroporation, cells were divided into 12-well plates and incubated in the transfection reagent with siRNA at $37^{\circ} \mathrm{C}$ in a humidified incubator with $5 \% \mathrm{CO}_{2}$ for $24 \mathrm{~h}$. Following the transfection procedure $10 \mu \mathrm{M}$ DENSPM was added into the plates.

Real-time quantitative PCR analysis. The total RNA was extracted using TRIzol (Invitrogen, USA) according to the manufacturer's protocol. The mRNA level of SSAT from the PCMV-SSAT or PCMV empty plasmid transfected LN229 cells, SSAT siRNA- or nonsense siRNA-transfected U87 cells, DENSPM-treated and untreated U87 and LN229 cells were quantified using the Applied Biosystems TaqMan method in conjunction with Assays-On-Demand (ABI Prism 7900 sequence detection system, Applied Biosystems,
A

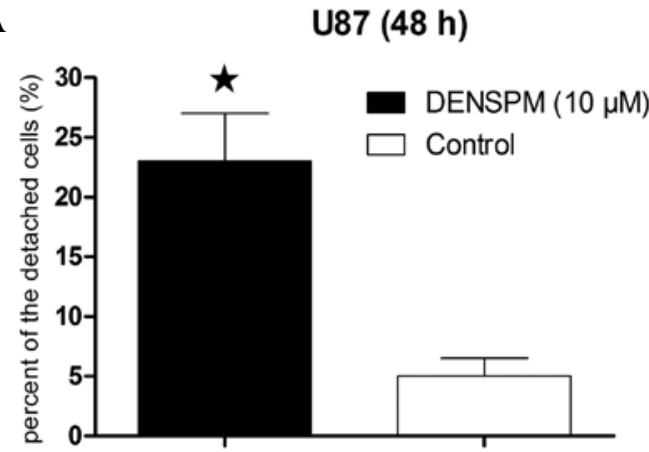

$\mathbf{B}$

LN229 (72 h)

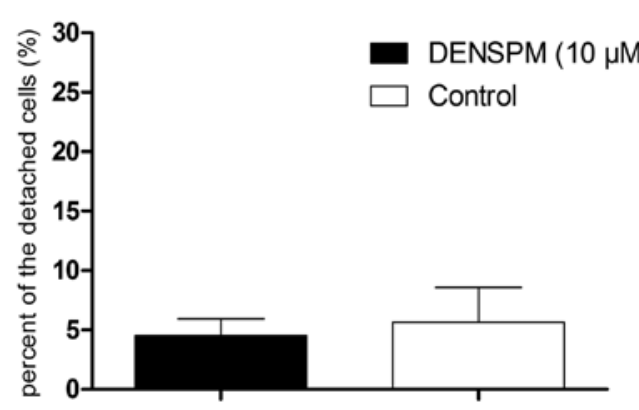

Figure 1. Cell detachment in both U87 and LN229 cells after treatment with DENSPM. (A) The percentage of floating U87 cells increased after incubation with DENSPM for $48 \mathrm{~h}$ relative to the control group $\left({ }^{\star} \mathrm{P}<0.05\right)$. (B) Cell detachment was not significant in LN229 cells treated with DENSPM for $72 \mathrm{~h}$.

Foster City, CA) based on the previous description (6). The results of real-time PCR were analyzed by the $\Delta \Delta \mathrm{CT}$ method: $\Delta \mathrm{CT}=\mathrm{CT}_{\text {selected gene }}-\mathrm{CT}_{\mathrm{GAPDH}}, \Delta \Delta \mathrm{CT}=\Delta \mathrm{CT}_{\text {therapy }}$ group $-\Delta \mathrm{CT}_{\text {control group }}, \mathrm{RV}$ (relative value) therapy group $=2^{-\Delta \Delta \mathrm{CT}}$, $\mathrm{RV}_{\text {control group }}=1$. The results of real-time PCR were presented as the ratio between the selected genes and GAPDH transcripts. The mean value of SSAT was calculated based on triplicate experiments.

Cell detachment examination. To evaluate the detachment status of cells treated with $10 \mu \mathrm{M}$ DENSPM or after PCMV-SSAT transfection or knockdown of SSAT, floating cells in the medium were collected first and then the adherent cells were collected by trypsinization. The percentage of detached cells was calculated by dividing the amount of the total floating cells and the trypsinized adherent cells by the number of the floating cells in the medium. The mean percentage of the detached cells was calculated based on triplicate experiments.

Cell viability assay. Cell viability was evaluated using the MTS assay (Promega Corporation, Madison, WI). For the MTS assay, we seeded 3,000 LN229 cells transfected with PCMV-SSAT or transfected SSAT siRNA per well in $100 \mu 1$ of medium in a 96-well plate. On the second day, varying concentrations of DENSPM were added to the wells. After $20 \mu \mathrm{l}$ of MTS solution had been added to each well and mixed, the cells were incubated at $37^{\circ} \mathrm{C}$ in the $5 \% \mathrm{CO}_{2}$ incubator. Absorbance at $490 \mathrm{~nm}$ was measured with a microplate reader (MRX, Danatech Laboratory, Houston, TX). All MTS assays were performed in triplicate for each treatment condition and experiments were repeated at least twice to confirm the consistency of results. 


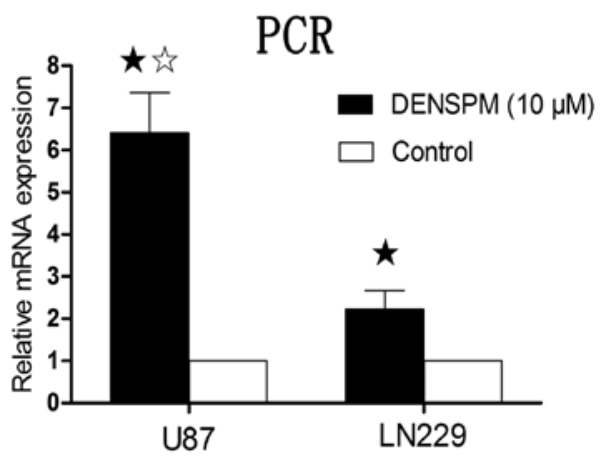

Figure 2. Expression of SSAT mRNA was higher in both U87 and LN229 cells after adding DENSPM compared with control groups $\left({ }^{\star} \mathrm{P}<0.05\right)$. The effect was significantly greater in U87 than LN229 cells ( $\left.{ }^{\circledR} \mathrm{P}<0.05\right)$.

Western blotting. LN229 cells transfected with PCMV-SSAT were lysed and homogenates were clarified by centrifugation at $12,000 \mathrm{xg}$ for $15 \mathrm{~min}$ at $4^{\circ} \mathrm{C}$. Supernatant samples were electrophoresed on $7.5 \%$ sodium dodecyl sulfate (SDS)polyacrylamide gels followed by transfer to polyvinylidene difluoride (PVDF) membranes (Millipore, USA). Incubation with primary polyclonal antibodies against integrin $\alpha 5$ (BD Biosciences, San Jose, CA), integrin $\beta 1$ (BD Biosciences), AKT, mTOR (Cell Signaling Technology, Danvers, MA), SSAT (Santa Cruz Biotechnology, Santa Cruz, CA) and actin (Sigma, St. Louis, MO) were performed at a dilution of 1:1,000 overnight at $4^{\circ} \mathrm{C}$. After washing, the membranes were incubated with secondary antibodies conjugated to biotin (Amersham Pharmacia Biotech, Piscataway, NJ) at a dilution of 1:3,000 for $30 \mathrm{~min}$ at room temperature. Reactions were developed with ECL or ECL plus (GE Healthcare, Buckinghamshire, UK). All Western blotting assays were performed in triplicate for each probed protein.

Statistical analysis. The SPSS16.0 software (SPSS, Chicago, IL) was used for statistical analysis. Differences in means were analyzed by the two-tailed $\mathrm{t}$-test, assuming unequal variances. All data are expressed as mean $\pm \mathrm{SD}$. A P-value $<0.05$ was considered significant.

\section{Results}

Cell detachment in DENSPM-treated glioblastoma cells is dependent on SSAT expression levels. After incubation with DENSPM $(10 \mu \mathrm{M})$ for $48 \mathrm{~h}$, the percentage of floating cells in U87 cells was $\sim 25 \%$ (Fig. 1A). While the incubation time of the same concentration of DENSPM in LN229 cells was extended up to $72 \mathrm{~h}$, this did not result in any significant cell detachment relative to control (Fig. 1B). The measurement of SSAT mRNA level with the real-time quantitative PCR revealed that $10 \mu \mathrm{M}$ of DENSPM induced significantly increased expression of SSAT in both U87 and LN229 cells. The induction of SSAT mRNA level was significantly higher in U87 cells than in LN229 when incubated for the same period of $24 \mathrm{~h}$ (Fig. 2).

Overexpression of SSAT in LN229 cells induced by transfection of the PCMV-SSAT results in increased cell detachment. The plasmids of PCMV-SSAT were successfully trans-
A

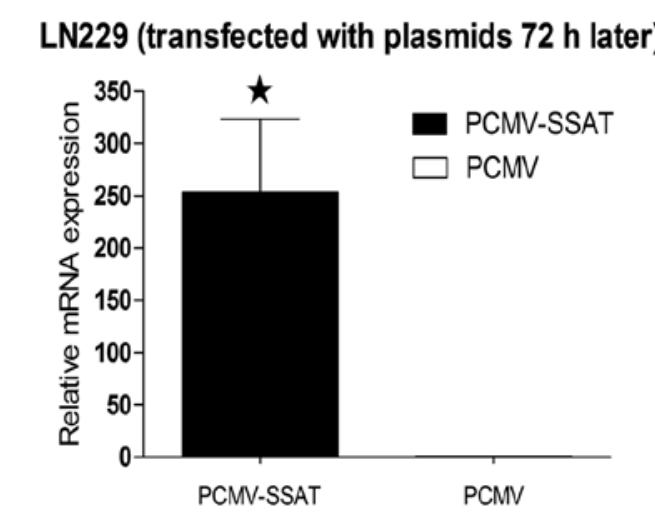

B

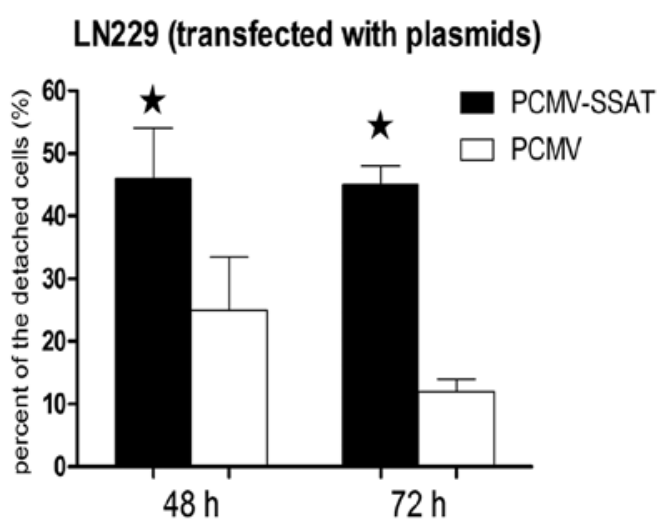

Figure 3. Overexpression of SSAT in LN229 cells transfected with PCMV-SSAT results in greater cell detachment. (A) SSAT mRNA was significantly elevated in PCMV-SSAT-LN229 cells after $72 \mathrm{~h}$ compared with PCMV-LN229 controls $\left({ }^{\star} \mathrm{P}<0.05\right)$. (B) The percentage of floating cells in PCMV-SSAT-LN229 cells increased significantly after $48 \mathrm{~h}$ and $72 \mathrm{~h}$ compared with the PCMV-LN229 control group $\left({ }^{\star} \mathrm{P}<0.05\right)$. There was no significant difference between 48 and $72 \mathrm{~h}$ within PCMV-SSAT transfected cells.

fected into LN229 cells. Real-time quantitative PCR was performed to confirm that SSAT mRNA was increased in the PCMV-SSAT-LN229 cells after $72 \mathrm{~h}$ (Fig. 3A). Then the percentage of the floating cells in the PCMV-SSAT-LN229 cells increased significantly after both $48 \mathrm{~h}$ and $72 \mathrm{~h}$ compared with cells transfected by PCMV empty plasmids. There was no significant difference in SSAT levels between $48 \mathrm{~h}$ and $72 \mathrm{~h}$ in transfected cells (Fig. 3B).

SSAT knockdown inhibits cell detachment in DENSPMtreated U87 cells. Knockdown of SSAT expression with siRNA was confirmed with real-time quantitative PCR (Fig. 4A). Detached cells were counted in DENSPM-treated and untreated U87 cells. A significant reduction in cell detachment was observed in SSAT siRNA-transfected U87 cells treated with DENSPM compared with the DENSPM alone treatment but was still significantly higher than in the control group (Fig. 4B).

Levels of SSAT affect DENSPM-mediated cytotoxicity in LN229 cells. Following successful SSAT upregulation by PCMV-SSAT transfection or knockdown by SSAT siRNA, an MTS assay was performed to determine the cytotoxicity of DENSPM in the LN229 cells with serial concentrations 
A

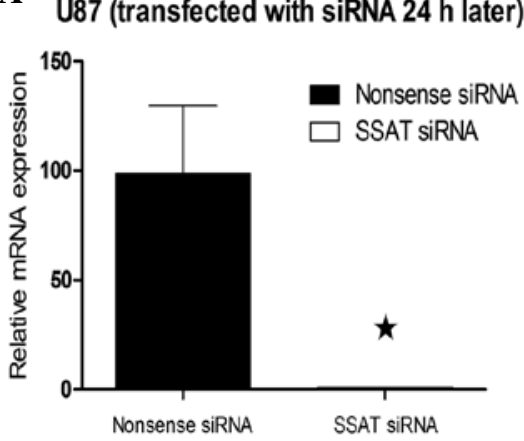

B

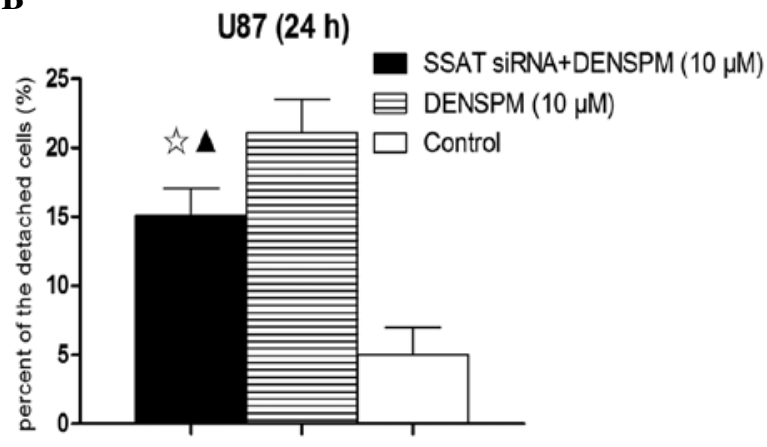

Figure 4. Knockdown of SSAT inhibited cell detachment in DENSPM-treated U87 cells. (A) SSAT mRNA was significantly reduced in SSAT siRNA transfected U87 cells after $24 \mathrm{~h}$ compared with the nonsense siRNA transfection group $\left({ }^{\star} \mathrm{P}<0.05\right)$. (B) The percentage of the floating cells in SSAT siRNA transfected U87 cells treated with DENSPM decreased significantly compared with DENSPM treatment alone ( ${ }^{*}$ P $\left.<0.05\right)$. However, the degree of cell detachment was still significantly higher compared to control group levels $\left({ }^{\wedge} \mathrm{P}<0.05\right)$.

A

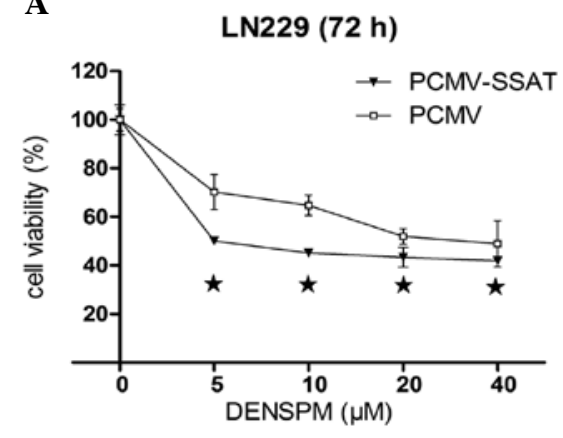

B

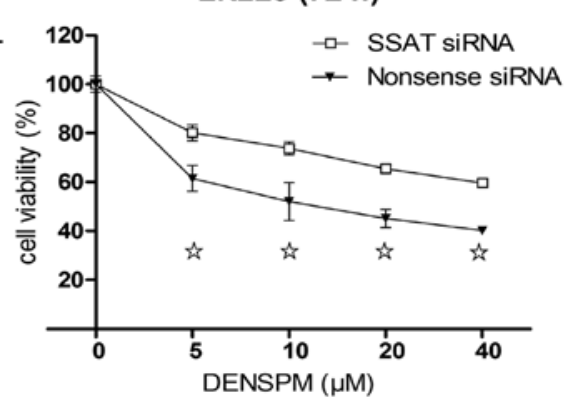

Figure 5. The level of SSAT expression was positively associated with DENSPM's cytotoxic effects in LN229 cell lines. (A) Cell viability was decreased in LN229 cells following induced SSAT mRNA expression $\left({ }^{\star} \mathrm{P}<0.05\right)$. (B) Conversely, cell viability was higher in SSAT siRNA-treated cells compared with the nonsense siRNA group ( $\left.{ }^{(} \mathrm{P}<0.05\right)$.

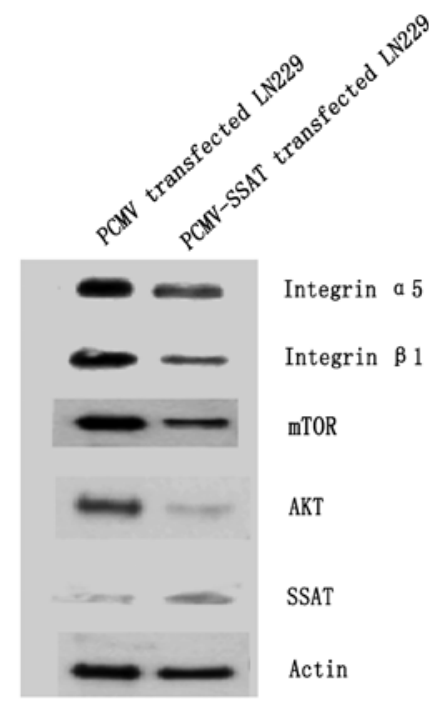

Figure 6. Reduced expression of anti-apoptosis related proteins AKT, mTOR and the adhesion-related proteins integrin $\alpha 5$, integrin $\beta 1$ in PCMVSSAT-LN229 cells.

of DENSPM. An elevation of SSAT resulted in enhanced cell killing in DENSPM-treated PCMV-SSAT-LN229 cells (Fig. 5A), while a mild attenuation of the DENSPM killing result was observed after the SSAT was turned down (Fig. 5B).
SSAT transfection induces degradation of anti-apoptosis and adhesion-related proteins. PCMV-SSAT was transfected in LN229 cells and confirmed by the real-time quantitative PCR. Expression of SSAT protein was found to be increased by $256.52 \%$ after transfection. The cell lysates were extracted after $48 \mathrm{~h}$ and Western blotting run for expression of antiapoptosis-related proteins AKT, mTOR and the adhesion related proteins integrin $\alpha 5$, integrin $\beta 1$. SSAT transfection reduced AKT, mTOR, integrin $\alpha 5$ and integrin $\beta 1$ expression by $35.90,77.06,79.91$ and $47.33 \%$, respectively (Fig. 6).

\section{Discussion}

Polyamines are essential requirements for eukaryotic cell growth. The metabolism of polyamines are frequently dysregulated in cancer, and the polyamine pathway is a main target for inhibiting the proliferation of carcinoma (2). Polyamine analogues resulting in polyamine depletion in cells are highly attractive chemotherapy targets for cancer. Polyamine analogues can enter into cells, compete with the natural polyamines for uptake but do not substitute for the natural polyamines in growth-related functions. This translates to polyamine analogue uptake in cancer cells resulting in inhibition of polyamine biosynthesis and polyamine catabolism (12). This is a key reason why polyamine analogues are more effective in cancer treatment than inhibitors of the polyamine 
biosynthesis enzymes. Significant induction of apoptosis by DENSPM was shown to occur in glioblastoma cell lines in our previous report (3). A preliminary exploration on the apoptosis-related signals induced by the polyamine analogue, DENSPM, has revealed that the AKT and the mTOR pathway play an important role in this process. SSAT expression has been noted to be induced after DENSPM application. However, its role in the apoptosis induction by a polyamine analog is far from clear $(3,8)$.

We first demonstrated that the rapid and significant increase in SSAT mRNA was associated with marked cell detachment and cell death in 2 glioblastoma cell lines. Porter and Casero have reported that the cell growth inhibition was caused by the excess induction of SSAT $(13,14)$. The SSAT activity in response to polyamine analogues has been shown to increase in many cell types $(15,16)$, but cannot be overexpressed in some cancer cell types $(9,17)$. DENSPM caused marked cell detachment in U87 cells and to a lesser extent in LN229 cells (Fig. 1). The mechanism responsible for the observed results of SSAT in different cell lines is not well understood. Our results support that the specificity of cell types determines the effect of SSAT cell adherence in response to DENSPM.

To evaluate whether elevated SSAT expression plays a key role in DENSPM-induced cell detachment and cell death in glioblastoma cells, the plasmid construct, PCMV-SSAT was developed to regulate the expression of SSAT in the tumor cells. Our results confirmed that the expression of SSAT was involved in determining the degree of the cell detachment and death consistent with findings by other groups. Hegardt and colleagues have reported that the elevation of SSAT activity in DENSPM-treated human breast cancer cells (L56Br-C1) induces whole cell detachment after $48 \mathrm{~h}$ incubation and a total cell death $72 \mathrm{~h}$ later (10). An overexpression of SSAT in kidney HEK 293 cells was also found to cause morphological shape change, the loss of cell anchorage, and the significant alteration of actin-containing filopodia which strongly suggested that the adhesion defect was associated with the high SSAT expression (11).

To further confirm that the induction of SSAT is associated with the cell adhesion, several adhesion-related proteins were examined. Integrins, control cell migration and proliferation through the interaction between neighboring cells and the surrounding extracellular matrix (ECM). Although several integrins have been recognized as key regulators in glioblastoma growth and angiogenesis (18), antagonism of the nonpeptidic $\alpha 5 \beta 1$ integrins has demonstrated anti-proliferative activity in glioblastoma cells and inhibition of cell adhesion in vitro and in vivo. Antagonism of $\alpha 5 \beta 1$ integrins are believed to inhibit angiogenesis impairing adhesion and migration of endothelial cells $(19,20)$. In our study, the expression of integrin $\alpha 5$ and integrin $\beta 1$ was down-regulated in PCMV-SSAT transfected LN229 cells detected by Western blotting. The low expression of both integrin $\alpha 5$ and integrin $\beta 1$ following SSAT induction correlates with increased cell detachment.

Increased SSAT expression was associated with apoptosis in the glioblastoma cells as demonstrated by decreased expression of anti-apoptosis-related signaling proteins AKT and mTOR. Both were deactivated in SSAT up-regulated glioblastomas and have been recognized as promising targets for therapeutic interventions (21-23). Moreover, simultaneous blockage of AKT and mTOR has been shown to markedly affect proliferative arrest in xenografted tumors (24). Interestingly, the overexpression of SSAT down-regulated AKT and mTOR expression in glioblastoma cells suggesting an effect on cell survival, since the DENSPM-inducing AKT and mTOR down-regulation has been proven to lead glioma cells to apoptosis (8).

Chen et al have reported that SSAT binds to integrin $\alpha 9$ and overexpression of SSAT increases integrin $\alpha 9 \beta 1$-mediated migration without decreasing cell viability. Furthermore, siRNA knockdown of SSAT inhibited this migration without affecting cell adhesion (25), which is in contrast to our findings. However, the association of SSAT with $\alpha 9 \beta 1$-mediated migration supports the notion of SSAT's role in cell adhesion and adhesion related apoptosis $(26,27)$.

In conclusion, our findings suggest that SSAT plays a partial role in adhesion related to cancer cell survival as evidenced by the subpopulation of cancer cells that responded to SSAT up-regulation. Further studies are warranted to confirm the function of SSAT in the treatment of glioblastoma.

\section{Acknowledgements}

We are grateful to Dr Eugen Park from Department of Critical Care in St. Michael's Hospital in Toronto for his invaluable suggestion and expert editorial review of our manuscript. This study was supported by the China National Natural Scientific Fund (30772230).

\section{References}

1. Elmore E, Stringer DE, Steele VE, Gerner EW and Redpath JL: Chemoprevention by difluoromethylornithine: correlation of an in vitro human cell assay with human clinical data for biomarker modulation. Anticancer Res 21: 1163-1165, 2001.

2. Gerner EW and Meyskens FL Jr: Polyamines and cancer: old molecules, new understanding. Nat Rev Cancer 4: 781-792, 2004.

3. Jiang R, Choi W, Khan A, et al: Activation of polyamine catabolism by $\mathrm{N}^{1}, \mathrm{~N}^{11}$-diethylnorspermine leads to cell death in glioblastoma. Int J Oncol 31: 431-440, 2007.

4. Pledgie A, Huang Y, Hacker A, et al: Spermine oxidase SMO(PAOh1), not N1-acetylpolyamine oxidase PAO, is the primary source of cytotoxic $\mathrm{H}_{2} \mathrm{O}_{2}$ in polyamine analogue-treated human breast cancer cell lines. J Biol Chem 280: 39843-39851, 2005.

5. Xu H, Chaturvedi R, Cheng Y, et al: Spermine oxidation induced by Helicobacter pylori results in apoptosis and DNA damage: implications for gastric carcinogenesis. Cancer Res 64: 8521-8525, 2004.

6. Choi W, Gerner EW, Ramdas L, et al: Combination of 5-fluorouracil and N1,N11-diethylnorspermine markedly activates spermidine/spermine N1-acetyltransferase expression, depletes polyamines, and synergistically induces apoptosis in colon carcinoma cells. J Biol Chem 280: 3295-3304, 2005.

7. Chen Y, Kramer DL, Diegelman P, Vujcic S and Porter CW: Apoptotic signaling in polyamine analogue-treated SK-MEL-28 human melanoma cells. Cancer Res 61: 6437-6444, 2001.

8. Jiang R, Choi W, Hu L, Gerner EW, Hamilton SR and Zhang W: Activation of polyamine catabolism by $\mathrm{N}^{1}, \mathrm{~N}^{11}$-diethylnorspermine alters the cellular localization of mTOR and downregulates mTOR protein level in glioblastoma cells. Cancer Biol Ther 6: 1644-1648, 2007.

9. Ha HC, Woster PM, Yager JD and Casero RA Jr: The role of polyamine catabolism in polyamine analogue-induced programmed cell death.Proc Natl Acad Sci USA 94: 11557-11562, 1997.

10. Hegardt C, Johannsson OT and Oredsson SM: Rapid caspasedependent cell death in cultured human breast cancer cells induced by the polyamine analogue $\mathrm{N}(1), \mathrm{N}(11)$-diethylnorspermine. Eur J Biochem 269: 1033-1039, 2002. 
11. Wang Z, Zahedi K, Barone S, et al: Overexpression of SSAT in kidney cells recapitulates various phenotypic aspects of kidney ischemia-reperfusion injury. J Am Soc Nephrol 15: 1844-1852, 2004.

12. Casero RA Jr and Marton LJ: Targeting polyamine metabolism and function in cancer and other hyperproliferative diseases. Nat Rev Drug Discov 6: 373-390, 2007.

13. Porter CW, Ganis B, Libby PR and Bergeron RJ: Correlations between polyamine analogue-induced increases in spermidine/spermine N1-acetyltransferase activity, polyamine pool depletion, and growth inhibition in human melanoma cell lines. Cancer Res 51: 3715-3720, 1991.

14. Casero RA Jr, Celano P, Ervin SJ, Wiest L and Pegg AE: High specific induction of spermidine/spermine $\mathrm{N}^{1}$-acetyltransferase in a human large cell lung carcinoma. Biochem J 270: 615-620, 1990.

15. Allen WL, McLean EG, Boyer J, et al: The role of spermidine/ spermine $\mathrm{N}^{1}$-acetyltransferase in determining response to chemotherapeutic agents in colorectal cancer cells. Mol Cancer Ther 6: $128-137,2007$.

16. Oredsson SM, Alm K, Dahlberg E, et al: Inhibition of cell proliferation and induction of apoptosis by $\mathrm{N}(1), \mathrm{N}(11)$ diethylnorspermine-induced polyamine pool reduction. Biochem Soc Trans 35: 405-409, 2007.

17. Casero RA Jr, Mank AR, Xiao L, Smith J, Bergeron RJ and Celano P: Steady-state messenger RNA and activity correlates with sensitivity to $\mathrm{N}^{1}, \mathrm{~N}^{12}$-bis(ethyl)spermine in human cell lines representing the major forms of lung cancer. Cancer Res 52: 5359-5363, 1992.

18. Uhm JH, Gladson CL and Rao JS: The role of integrins in the malignant phenotype of gliomas. Front Biosci 4: D188-D199, 1999.

19. Maglott A, Bartik P, Cosgun S, et al: The small alpha5beta1 integrin antagonist, SJ749, reduces proliferation and clonogenicity of human astrocytoma cells. Cancer Res 66: 6002-6007, 2006.
20. Farber K, Synowitz M, Zahn G, et al: An alpha5beta1 integrin inhibitor attenuates glioma growth. Mol Cell Neurosci 39: $579-585,2008$.

21. Kesari S, Ramakrishna N, Sauvageot C, Stiles CD and Wen PY: Targeted molecular therapy of malignant gliomas. Curr Neurol Neurosci Rep 5: 186-197, 2005.

22. Capdevila J,Salazar R, Halperin I, Abad A and Yao JC: Innovations therapy: mammalian target of rapamycin (mTOR) inhibitors for the treatment of neuroendocrine tumors. Cancer Metastasis Rev 30: 27-34, 2011.

23. Martelli AM, Evangelisti C, Follo MY, et al: Targeting the phosphatidylinositol 3-kinase/akt/mammalian target of rapamycin signaling network in cancer stem cells. Curr Med Chem 18: 2715-2726, 2011

24. Fan QW, Knight ZA, Goldenberg DD, et al: A dual PI3-kinase/ mTOR inhibitor reveals emergent efficacy in glioma. Cancer Cell 9: 341-349, 2006.

25. Chen C, Young BA, Coleman CS, Pegg AE and Sheppard D: Spermidine/spermine $\mathrm{N}^{1}$-acetyltransferase specifically binds to the integrin alpha9 subunit cytoplasmic domain and enhances cell migration. J Cell Biol 167: 161-170, 2004.

26. Ross EA, Douglas MR, Wong SH, et al: Interaction between integrin alpha9beta1 and vascular cell adhesion molecule-1 (VCAM-1) inhibits neutrophil apoptosis. Blood 107: 1178-1183, 2006.

27. Taooka Y, Chen J, Yednock T and Sheppard D: The integrin alpha9beta1 mediates adhesion to activated endothelial cells and transendothelial neutrophil migration through interaction with vascular cell adhesion molecule-1. J Cell Biol 145: 413-420, 1999. 